\title{
KZ Characteristic Variety as the Zero Set of Classical Calogero-Moser Hamiltonians
}

Evgeny MUKHIN ${ }^{\dagger}$, Vitaly TARASOV ${ }^{\dagger \ddagger}$ and Alexander VARCHENKO $\S$

${ }^{\dagger}$ Department of Mathematical Sciences, Indiana University - Purdue University Indianapolis, 402 North Blackford St, Indianapolis, IN 46202-3216, USA

E-mail: mukhin@math.iupui.edu,vtarasov@math.iupui.edu

$\ddagger$ St. Petersburg Branch of Steklov Mathematical Institute, Fontanka 27, St. Petersburg, 191023, Russia

E-mail: vt@pdmi.ras.ru

$\S$ Department of Mathematics, University of North Carolina at Chapel Hill, Chapel Hill, NC 27599-3250, USA

E-mail: anv@email.unc.edu

Received June 09, 2012, in final form October 04, 2012; Published online October 16, 2012

http://dx.doi.org/10.3842/SIGMA.2012.072

Abstract. We discuss a relation between the characteristic variety of the KZ equations and the zero set of the classical Calogero-Moser Hamiltonians.

Key words: Gaudin Hamiltonians; Calogero-Moser system; Wronski map

2010 Mathematics Subject Classification: 82B23; 17B80

\section{Statement of results}

\section{$1.1 \quad$ Motivation}

This paper is motivated by the Givental and Kim observation [10] that the characteristic variety of the quantum differential equation of a flag variety is a Lagrangian variety of the classical Toda lattice. The quantum differential equation is a system of differential equations $\hbar \partial_{i} \psi=b_{i} \circ \psi$, $i=1, \ldots, r$, defined by the quantum multiplication $\circ$ and depending on a parameter $\hbar$. The system defines a flat connection for all nonzero values of $\hbar$. Givental and Kim, in particular, observe that the characteristic variety of this system is the Lagrangian variety of the classical Toda lattice, defined by equating to zero the first integrals of the Toda lattice.

In this paper we describe a similar relation between the $\mathrm{KZ}$ equation and the classical Calogero-Moser system. On numerous relations between the KZ equations and quantum Calogero-Moser systems see $[2,3,6,7,14]$.

\subsection{Classical Calogero-Moser system}

Fix an integer $n \geqslant 2$. Denote $\Delta=\left\{\boldsymbol{z}=\left(z_{1}, \ldots, z_{n}\right) \in \mathbb{C}^{n} \mid z_{a}=z_{b}\right.$ for some $\left.a \neq b\right\}$, the union of diagonals. Consider the cotangent bundle $T^{*}\left(\mathbb{C}^{n}-\Delta\right)$ with symplectic form $\omega=\sum_{a=1}^{n} d p_{a} \wedge d z_{a}$, where $p_{1}, \ldots, p_{n}$ are coordinates on fibers. The classical Calogero-Moser system on $T^{*}\left(\mathbb{C}^{n}-\Delta\right)$ is defined by the Hamiltonian

$$
\mathcal{H}=\sum_{a=1}^{n} p_{a}^{2}-\sum_{1 \leqslant a<b \leqslant n} \frac{2}{\left(z_{a}-z_{b}\right)^{2}}
$$


The system is completely integrable. For

$$
Q=\left(\begin{array}{ccccc}
p_{1} & \frac{1}{z_{1}-z_{2}} & \frac{1}{z_{1}-z_{3}} & \cdots & \frac{1}{z_{1}-z_{n}} \\
\frac{1}{z_{2}-z_{1}} & p_{2} & \frac{1}{z_{2}-z_{3}} & \cdots & \frac{1}{z_{2}-z_{n}} \\
\ldots & \ldots & \ldots & \ldots & \ldots \\
\frac{1}{z_{n}-z_{1}} & \frac{1}{z_{n}-z_{2}} & \frac{1}{z_{n}-z_{3}} & \ldots & p_{n}
\end{array}\right),
$$

let $\operatorname{det}(u-Q)=u^{n}-Q_{1} u^{n-1}+\cdots \pm Q_{n}$ be the characteristic polynomial. Then $Q_{1}, \ldots, Q_{n}$ is a complete list of commuting first integrals, and $\mathcal{H}=Q_{1}^{2}-Q_{2}$.

We will be interested in the subvariety $L_{0} \subset T^{*}\left(\mathbb{C}^{n}-\Delta\right)$ defined by the equations

$$
L_{0}=\left\{(\boldsymbol{z}, \boldsymbol{p}) \in T^{*}\left(\mathbb{C}^{n}-\Delta\right) \mid Q_{a}(\boldsymbol{z}, \boldsymbol{p})=0, a=1, \ldots, n\right\} .
$$

Theorem 1.1 ([23]). For any $n$, the subvariety $L_{0}$ is smooth and Lagrangian.

See propositions in Section 6 of [23]. Another proof of Theorem 1.1 will be given in Section 2.4.

\subsection{Gaudin Hamiltonians and $\mathrm{KZ}$ characteristic variety}

Fix an integer $N \geqslant 2$. Denote $V=\mathbb{C}^{N}$ the vector representation of the Lie algebra $\mathfrak{g l}_{N}$. The Hamiltonians of the quantum Gaudin model are the linear operators $H_{1}, \ldots, H_{n}$ on the space $V^{\otimes n}$,

$$
H_{a}(\boldsymbol{z})=\sum_{i, j=1}^{N} \sum_{b \neq a} \frac{e_{i j}^{(a)} e_{j i}^{(b)}}{z_{a}-z_{b}},
$$

where $e_{i j}$ are the standard generators of $\mathfrak{g l}_{N}, e_{i j}^{(a)}$ is the image of $1^{\otimes(a-1)} \otimes e_{i j} \otimes 1^{\otimes(n-a)}$, and $z_{1}, \ldots, z_{n}$ are distinct complex numbers, see [9]. The operators commute, $\left[H_{a}(\boldsymbol{z}), H_{b}(\boldsymbol{z})\right]=0$ for all $a, b$. The operators commute with the $\mathfrak{g l}_{N}$-action on $V^{\otimes n}$.

Let $\boldsymbol{\lambda}=\left(\lambda_{1}, \ldots, \lambda_{N}\right) \in \mathbb{Z}_{\geqslant 0}^{N}$ be a partition of $n$ with at most $N$ parts, $\lambda_{1} \geqslant \cdots \geqslant \lambda_{N}$, $|\boldsymbol{\lambda}|=\lambda_{1}+\cdots+\lambda_{N}=n$. Denote

$$
\text { Sing } V^{\otimes n}[\boldsymbol{\lambda}]=\left\{v \in V^{\otimes n} \mid e_{i i} v=\lambda_{i} v, i=1, \ldots, N ; e_{i j} v=0 \text { for all } i<j\right\},
$$

the subspace of singular vectors of weight $\boldsymbol{\lambda}$. The Gaudin Hamiltonians preserve Sing $V^{\otimes n}[\boldsymbol{\lambda}]$. We define the spectral variety of the Gaudin model on Sing $V^{\otimes n}[\boldsymbol{\lambda}]$,

$$
\operatorname{Spec}_{N, \boldsymbol{\lambda}}=\left\{(\boldsymbol{z}, \boldsymbol{p}) \in T^{*}\left(\mathbb{C}^{n}-\Delta\right) \mid \exists v \in \operatorname{Sing} V^{\otimes n}[\boldsymbol{\lambda}] \text { with } H_{a}(\boldsymbol{z}) v=p_{a} v, a=1, \ldots, n\right\} .
$$

The spectral variety is a Lagrangian subvariety of $T^{*}\left(\mathbb{C}^{n}-\Delta\right)$, see, for example, Proposition 1.5 in $[20]$.

The Gaudin Hamiltonians are the right hand sides of the KZ equations,

$$
\kappa \partial_{z_{i}} I(\boldsymbol{z})=H_{i}(\boldsymbol{z}) I(\boldsymbol{z}), \quad i=1, \ldots, n,
$$

where $\kappa \in \mathbb{C}^{\times}$is a parameter. The spectral variety $\operatorname{Spec}_{N, \boldsymbol{\lambda}}$ is, by definition, the characteristic variety of the $\kappa$-dependent $D$-module defined by the KZ equations with values $\operatorname{Sing} V^{\otimes n}[\boldsymbol{\lambda}]$.

Example. If $\boldsymbol{\lambda}=(n, 0, \ldots, 0)$, then $\operatorname{Spec}_{N, \boldsymbol{\lambda}}$ is given by the equations $p_{a}=\sum_{b \neq a}\left(z_{a}-z_{b}\right)^{-1}, a=1$, $\ldots, n$. If $N=n$ and $\boldsymbol{\lambda}=(1, \ldots, 1)$, then $\operatorname{Spec}_{N, \boldsymbol{\lambda}}$ is given by the equations $p_{a}=-\sum_{b \neq a}\left(z_{a}-z_{b}\right)^{-1}$, $a=1, \ldots, n$. 


\section{Theorem 1.2.}

(i) The variety $\operatorname{Spec}_{N, \boldsymbol{\lambda}}$ does not depend on $N$. Namely, consider $\boldsymbol{\lambda}$ as a partition of $n$ with at most $N+1$ parts. Then $\operatorname{Spec}_{N, \boldsymbol{\lambda}}=\operatorname{Spec}_{N+1, \boldsymbol{\lambda}}$. From now on we denote $\operatorname{Spec}_{N, \boldsymbol{\lambda}}$ by $\operatorname{Spec}_{\boldsymbol{\lambda}}$.

(ii) For any $n$, the variety $L_{0}$ is the disjoint union of the varieties $\mathrm{Spec}_{\boldsymbol{\lambda}}$, where the union is over all partitions $\boldsymbol{\lambda}$ of $n$.

By Theorem 1.1, each $\mathrm{Spec}_{\boldsymbol{\lambda}}$ is smooth and Lagrangian.

Part $(i)$ of Theorem 1.2 is proved in Section 2.2, and part (ii) is proved in Section 2.4.

\subsection{Master function generates $\operatorname{Spec}_{\lambda}$}

Let $\boldsymbol{\lambda}$ be a partition of $n$ with at most $N$ parts. Denote $l_{a}=\sum_{b=a+1}^{N} \lambda_{b}, a=1, \ldots, N-1$. Denote $l=l_{1}+\cdots+l_{N-1}$. Consider the set of $l$ variables

$$
\boldsymbol{t}=\left(t_{1}^{(1)}, \ldots, t_{l_{1}}^{(1)}, \ldots, t_{1}^{(N-1)}, \ldots, t_{l_{N-1}}^{(N-1)}\right)
$$

and the affine space $\mathbb{C}^{n} \times \mathbb{C}^{l}$ with coordinates $\boldsymbol{z}, \boldsymbol{t}$. The function $\Phi_{N, \boldsymbol{\lambda}}: \mathbb{C}^{n} \times \mathbb{C}^{l} \rightarrow \mathbb{C}$,

$$
\begin{aligned}
\Phi_{N, \boldsymbol{\lambda}}(\boldsymbol{z}, \boldsymbol{t})= & \sum_{1 \leqslant a<b \leqslant n} \log \left(z_{a}-z_{b}\right)-\sum_{a=1}^{n} \sum_{i=1}^{l_{a}} \log \left(t_{i}^{(1)}-z_{a}\right) \\
& +2 \sum_{k=1}^{N-1} \sum_{1 \leqslant i<j \leqslant l_{k}} \log \left(t_{i}^{(k)}-t_{j}^{(k)}\right)-\sum_{k=0}^{N-2} \sum_{i=1}^{l_{k}} \sum_{j=1}^{l_{k+1}} \log \left(t_{i}^{(k)}-t_{j}^{(k+1)}\right)
\end{aligned}
$$

is called the master function, see [21, 22].

The master function depends on $\boldsymbol{\lambda}$, but not on $N$. Namely, consider $\boldsymbol{\lambda}$ as a partition of $n$ with at most $N+1$ parts. Then $\Phi_{N, \boldsymbol{\lambda}}=\Phi_{N+1, \boldsymbol{\lambda}}$. From now on we denote $\Phi_{N, \boldsymbol{\lambda}}$ by $\Phi_{\boldsymbol{\lambda}}$.

Critical points of $\Phi_{\boldsymbol{\lambda}}$ with respect to $\boldsymbol{t}$ are given by the equation $d_{\boldsymbol{t}} \Phi_{\boldsymbol{\lambda}}=0$. Denote by Crit $\boldsymbol{\lambda}_{\boldsymbol{\lambda}}$ the critical set of $\Phi_{\boldsymbol{\lambda}}$ with respect to $\boldsymbol{t}$,

$$
\text { Crit }_{\boldsymbol{\lambda}}=\left\{(\boldsymbol{z}, \boldsymbol{t}) \in \mathbb{C}^{n} \times \mathbb{C}^{l} \mid d_{\boldsymbol{t}} \Phi_{\boldsymbol{\lambda}}(\boldsymbol{z}, \boldsymbol{t})=0\right\} .
$$

This is an algebraic subset of the domain of $\mathbb{C}^{n} \times \mathbb{C}^{l}$, where the master function is a regular (multivalued) function. Denote by $L_{\boldsymbol{\lambda}} \subset T^{*}\left(\mathbb{C}^{n}-\Delta\right)$ the image of the map

$$
\text { Crit }_{\boldsymbol{\lambda}} \rightarrow T^{*}\left(\mathbb{C}^{n}-\Delta\right), \quad(\boldsymbol{z}, \boldsymbol{t}) \mapsto(\boldsymbol{z}, \boldsymbol{p}), \quad \text { where } \quad p_{a}=\frac{\partial \Phi_{\boldsymbol{\lambda}}}{\partial z_{a}}(\boldsymbol{z}, \boldsymbol{t}), \quad a=1, \ldots, n .
$$

Theorem 1.3. For any $n$ and a partition $\boldsymbol{\lambda}$ of $n$, we have $L_{\boldsymbol{\lambda}} \subset \mathrm{Spec}_{\boldsymbol{\lambda}}$ and $\mathrm{Spec}_{\boldsymbol{\lambda}}$ is the closure of $L_{\boldsymbol{\lambda}}$ in $T^{*}\left(\mathbb{C}^{n}-\Delta\right)$.

Theorem 1.3 is proved in Section 2.1.

\subsection{Calogero-Moser space $C_{n}$ and cotangent bundle $T^{*}\left(\mathbb{C}^{n}-\Delta\right)$}

The Calogero-Moser system has singularities if some of $z_{1}, \ldots, z_{n}$ coincide. These singularities can be resolved and the Calogero-Moser system can be lifted by the map $\xi$ given by (1.4) below to a regular completely integrable Hamiltonian system on the Calogero-Moser space $C_{n}$, see [13].

Denote

$$
\widetilde{C}_{n}=\left\{(Z, Q) \in \mathfrak{g l}_{n} \times \mathfrak{g l}_{n} \mid \operatorname{rank}([Z, Q]+1)=1\right\} .
$$


The group $G L_{n}$ of complex invertible matrices acts on $\widetilde{C}_{n}$ by simultaneous conjugation. The action is free and proper, see [23]. The quotient space $C_{n}$ is called the $n$-th Calogero-Moser space. The Calogero-Moser space $C_{n}$ is a smooth affine variety of dimension $2 n$, see [23].

The group $S_{n}$ freely acts on $\mathbb{C}^{n}-\Delta$ by permuting coordinates. The action lifts to a free action on $T^{*}\left(\mathbb{C}^{n}-\Delta\right)$. Define the map

$$
\xi: T^{*}\left(\mathbb{C}^{n}-\Delta\right) \rightarrow T^{*}\left(\mathbb{C}^{n}-\Delta\right) / S_{n} \rightarrow C_{n}
$$

by the rule: $(\boldsymbol{z}, \boldsymbol{p})$ is mapped to $(Z, Q)$, where $Z=\operatorname{diag}\left(z_{1}, \ldots, z_{n}\right)$ and $Q$ is defined by (1.1). The map $\xi$ induces an embedding $T^{*}\left(\mathbb{C}^{n}-\Delta\right) / S_{n} \rightarrow C_{n}$ whose image is Zariski open in $C_{n}$.

Set $\mathbb{C}^{(n)}=\mathbb{C}^{n} / S_{n}$ and let $\operatorname{spec}(X) \in \mathbb{C}^{(n)}$ stand for the point given by the eigenvalues of a square matrix $X$. The canonical map

$$
\pi: C_{n} \rightarrow \mathbb{C}^{(n)} \times \mathbb{C}^{(n)}, \quad(Z, Q) \mapsto(\operatorname{spec}(Z), \operatorname{spec}(Q)),
$$

is a finite map of degree $n$ !, see [4]. This map and its fiber over $0 \times 0$ were studied, for example, in $[4,8,11]$.

Let $C_{n}^{0}$ be the subvariety $\pi^{-1}\left(\mathbb{C}^{(n)} \times 0\right) \subset C_{n}$. Identifying $\mathbb{C}^{(n)} \times 0$ with $\mathbb{C}^{(n)}$ we get a map

$$
\pi^{0}: C_{n}^{0} \rightarrow \mathbb{C}^{(n)}, \quad(Z, Q) \mapsto \operatorname{spec}(Z),
$$

induced by $\pi$. We will describe $\pi^{0}$ in Section 1.8 .

\subsection{Wronski map}

For a partition $\boldsymbol{\lambda}$ of $n$, introduce $\widetilde{\boldsymbol{\lambda}}=\left\{\widetilde{\lambda}_{1}, \ldots, \widetilde{\lambda}_{n}\right\}$ by $\widetilde{\lambda}_{i}=\lambda_{i}+n-i$. Denote

$$
f_{i}(u)=u^{\widetilde{\lambda}_{i}}+\sum_{\substack{j=1 \\ \tilde{\lambda}_{i}-j \notin \tilde{\lambda}}}^{\tilde{\lambda}_{i}} f_{i j} u^{\widetilde{\lambda}_{i}-j}, \quad i=1, \ldots, n .
$$

Denote $X_{\boldsymbol{\lambda}}$ the $n$-dimensional affine space of $n$-tuples $\left\{f_{1}, \ldots, f_{n}\right\}$ of such polynomials. The polynomial algebra $\mathbb{C}\left[X_{\boldsymbol{\lambda}}\right]=\mathbb{C}\left[f_{i j}, i=1, \ldots, n, j \in\left\{1, \ldots, \widetilde{\lambda}_{i}\right\}, \widetilde{\lambda}_{i}-j \notin \widetilde{\boldsymbol{\lambda}}\right]$ is the algebra of regular functions on $X_{\lambda}$.

If $\boldsymbol{z}=\left(z_{1}, \ldots, z_{n}\right)$ are coordinates on $\mathbb{C}^{n}$, then $\boldsymbol{\sigma}=\left(\sigma_{1}, \ldots, \sigma_{n}\right)$, where $\sigma_{a}$ is the $a$-th elementary symmetric function of $z_{1}, \ldots, z_{n}$, are coordinates on $\mathbb{C}^{(n)}=\mathbb{C}^{n} / S_{n}$.

For arbitrary functions $g_{1}(u), \ldots, g_{n}(u)$, introduce the Wronskian determinant by the formula

$$
\operatorname{Wr}\left(g_{1}(u), \ldots, g_{n}(u)\right)=\operatorname{det}\left(\begin{array}{cccc}
g_{1}(u) & g_{1}^{\prime}(u) & \ldots & g_{1}^{(n-1)}(u) \\
g_{2}(u) & g_{2}^{\prime}(u) & \ldots & g_{2}^{(n-1)}(u) \\
\ldots & \ldots & \ldots & \ldots \\
g_{n}(u) & g_{n}^{\prime}(u) & \ldots & g_{n}^{(n-1)}(u)
\end{array}\right)
$$

We have

$$
\operatorname{Wr}\left(f_{1}(u), \ldots, f_{n}(u)\right)=\prod_{1 \leqslant i<j \leqslant n}\left(\widetilde{\lambda}_{j}-\widetilde{\lambda}_{i}\right) \cdot\left(u^{n}+\sum_{a=1}^{n}(-1)^{a} W_{a} u^{n-a}\right)
$$

with $W_{1}, \ldots, W_{n} \in \mathbb{C}\left[X_{\boldsymbol{\lambda}}\right]$. Define an algebra homomorphism

$$
\mathcal{W}_{\boldsymbol{\lambda}}: \mathbb{C}\left[\mathbb{C}^{(n)}\right] \rightarrow \mathbb{C}\left[X_{\boldsymbol{\lambda}}\right], \quad \sigma_{a} \mapsto W_{a}
$$

The corresponding map $\mathrm{Wr}_{\boldsymbol{\lambda}}: X_{\boldsymbol{\lambda}} \rightarrow \mathbb{C}^{(n)}$ is called the Wronski map. 
Denote

$$
X_{\boldsymbol{\lambda}}^{0}=X_{\boldsymbol{\lambda}} \cap \mathrm{Wr}_{\boldsymbol{\lambda}}^{-1}\left(\left(\mathbb{C}^{n}-\Delta\right) / S_{n}\right) .
$$

Irreducible representations of the symmetric group $S_{n}$ are labeled by partitions $\boldsymbol{\lambda}$ of $n$. Denote by $d_{\boldsymbol{\lambda}}$ the dimension of the irreducible representation corresponding to $\boldsymbol{\lambda}$. The Wronski map is a finite map of degree $d_{\boldsymbol{\lambda}}$, see, for example, [18].

\subsection{Universal differential operator on $\boldsymbol{X}_{\lambda}$}

Given an $n \times n$ matrix $A$ with possibly noncommuting entries $a_{i j}$, we define the row determinant to be

$$
\operatorname{rdet} A=\sum_{\sigma \in S_{n}}(-1)^{\sigma} a_{1 \sigma(1)} a_{2 \sigma(2)} \cdots a_{n \sigma(n)}
$$

Let $x=\left(f_{1}, \ldots, f_{n}\right)$ be a point of $X_{\boldsymbol{\lambda}}$. Define the differential operator $\mathcal{D}_{\boldsymbol{\lambda}, x}$ by

$$
\mathcal{D}_{\boldsymbol{\lambda}, x}=\prod_{1 \leqslant i<j \leqslant n}\left(\widetilde{\lambda}_{j}-\widetilde{\lambda}_{i}\right)^{-1} \cdot \operatorname{rdet}\left(\begin{array}{cccc}
f_{1}(u) & f_{1}^{\prime}(u) & \ldots & f_{1}^{(n)}(u) \\
f_{2}(u) & f_{2}^{\prime}(u) & \ldots & f_{2}^{(n)}(u) \\
\ldots & \ldots & \ldots & \ldots \\
1 & \partial & \ldots & \partial^{n}
\end{array}\right),
$$

where $\partial=d / d u$. It is a differential operator in variable $u$,

$$
\mathcal{D}_{\boldsymbol{\lambda}, x}=\sum_{0 \leqslant i \leqslant j \leqslant n} P_{i j}(x) u^{n-j} \partial^{n-i}
$$

with $P_{i j} \in \mathbb{C}\left[X_{\boldsymbol{\lambda}}\right]$. By formulae (2.11) and (2.3) in [18], we have

$$
\sum_{i=1}^{n} P_{i i} \prod_{j=i+1}^{n}(s+j)=\prod_{j=1}^{n}\left(s-\lambda_{j}+j\right),
$$

where $s$ is an independent formal variable.

Let $x \in X_{\boldsymbol{\lambda}}^{0}$. Fix $\boldsymbol{z}_{x}=\left(z_{1, x}, \ldots, z_{n, x}\right) \in \mathbb{C}^{n}$ corresponding to $\operatorname{Wr}_{\boldsymbol{\lambda}}(x) \in \mathbb{C}^{(n)}$. Then

$$
\begin{aligned}
\mathcal{D}_{\boldsymbol{\lambda}, x}= & \prod_{a=1}^{n}\left(u-z_{a, x}\right)\left(\partial^{n}-\sum_{a=1}^{n} \frac{1}{u-z_{a, x}} \partial^{n-1}\right. \\
& \left.+\sum_{a=1}^{n} \frac{1}{u-z_{a, x}}\left(-p_{a, x}+\sum_{b \neq a} \frac{1}{z_{a, x}-z_{b, x}}\right) \partial^{n-2}+\cdots\right)
\end{aligned}
$$

for suitable numbers $\boldsymbol{p}_{x}=\left(p_{1, x}, \ldots, p_{n, x}\right)$, see Lemma 3.1 in [16].

Lemma 1.4. The map

$$
\psi_{\boldsymbol{\lambda}}: X_{\boldsymbol{\lambda}}^{0} \rightarrow T^{*}\left(\mathbb{C}^{n}-\Delta\right) / S_{n}, \quad x \mapsto\left(\boldsymbol{z}_{x}, \boldsymbol{p}_{x}\right),
$$

is an embedding whose image is $\operatorname{Spec}_{\boldsymbol{\lambda}} / S_{n}$.

Lemma 1.4 is proved in Section 2.3. 


\subsection{Description of $\pi^{0}$}

\section{Theorem 1.5.}

(i) The irreducible components of the subvariety $C_{n}^{0} \subset C_{n}$ are naturally labeled by partitions $\boldsymbol{\lambda}$ of $n, C_{n}^{0}=\cup_{\boldsymbol{\lambda}} C_{\boldsymbol{\lambda}}^{0}$, where $C_{\boldsymbol{\lambda}}^{0}$ is the closure of $\xi\left(\operatorname{Spec}_{\boldsymbol{\lambda}}\right)$ in $C_{n}$.

(ii) For any $\boldsymbol{\lambda}$, the equations $Q_{a}=0, a=1, \ldots, n$, define $C_{\boldsymbol{\lambda}}^{0}$ in $C_{n}$ with multiplicity $d_{\boldsymbol{\lambda}}$.

(iii) The irreducible components of $C_{n}^{0}$ do not intersect. Each component is an n-dimensional submanifold of $C_{n}$ isomorphic to an n-dimensional affine space, [23].

(iv) Let $\boldsymbol{\lambda}$ be a partition of $n$. Then there is an embedding $\varphi_{\boldsymbol{\lambda}}: X_{\boldsymbol{\lambda}} \rightarrow C_{n}^{0}$ whose image is $C_{\boldsymbol{\lambda}}^{0}$ and such that the following diagram is commutative:

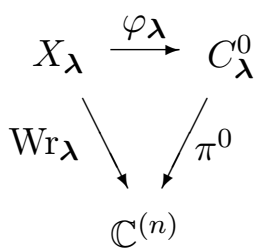

C.f. the statements $(i)-(i i i)$ with results in [8].

The map $\varphi_{\boldsymbol{\lambda}}$ is given by the following construction. The restriction of $\varphi_{\boldsymbol{\lambda}}$ to $X_{\boldsymbol{\lambda}}^{0}$ is the composition $\xi \circ \psi_{\boldsymbol{\lambda}}$, where $\xi$ is given by (1.4). This map extends from $X_{\boldsymbol{\lambda}}^{0}$ to an embedding $X_{\boldsymbol{\lambda}} \rightarrow C_{n}^{0}$, see Section 2.5.

Parts $(i)$ and $(i i)$ of Theorem 1.5 are proved in Section 2.4. Parts (iii) and (iv) of Theorem 1.5 are proved in Section 2.5.

Remark. It follows from Theorem 1.5 that $\pi^{-1}(0 \times 0)$ consists of points labeled by partitions and the multiplicity of the point corresponding to a partition $\boldsymbol{\lambda}$ equals $\left(d_{\boldsymbol{\lambda}}\right)^{2}$.

The fact that the points of $\pi^{-1}(0 \times 0)$ are labeled by partitions was explained in [4]. The fact that the multiplicity equals $\left(d_{\boldsymbol{\lambda}}\right)^{2}$ was formulated in [4] as Conjecture 17.14 and proved in [8].

\section{Proofs}

\subsection{Proof of Theorem 1.3}

Assume that a point $\boldsymbol{z}=\left(z_{1}, \ldots, z_{n}\right)$ has distinct coordinates. The Bethe ansatz construction assigns an eigenvector $\omega(\boldsymbol{z}, \boldsymbol{t})$ of Gaudin Hamiltonians $H_{a}(\boldsymbol{z})$ on Sing $V^{\otimes n}[\boldsymbol{\lambda}]$ to a critical point $(\boldsymbol{z}, \boldsymbol{t})$ of the master function $\Phi_{\boldsymbol{\lambda}}(\boldsymbol{z}, \boldsymbol{t})$, see $[1,12,19,20]$,

$$
H_{a}(\boldsymbol{z}) \omega(\boldsymbol{z}, \boldsymbol{t})=\frac{\partial \Phi_{\boldsymbol{\lambda}}}{\partial z_{a}}(\boldsymbol{z}, \boldsymbol{t}) \omega(\boldsymbol{z}, \boldsymbol{t}), \quad a=1, \ldots, n
$$

Formula (2.1) shows that $L_{\boldsymbol{\lambda}} \subset \operatorname{Spec}_{\boldsymbol{\lambda}}$. By Theorem 6.1 in [19] the Bethe vectors form a basis of Sing $V^{\otimes n}[\boldsymbol{\lambda}]$ for generic $\boldsymbol{z} \in \mathbb{C}^{n}-\Delta$. This proves Theorem 1.3.

\subsection{Proof of part (i) of Theorem 1.2}

It is easy to see that Sing $V^{\otimes n}[\boldsymbol{\lambda}]$ and the action on it of the Hamiltonians $H_{a}(\boldsymbol{z})$ do not depend on $N$. Hence, $\operatorname{Spec}_{N, \boldsymbol{\lambda}}$ does not depend on $N$.

Another (less straightforward) proof of part $(i)$ follows from formula (2.1) and the fact that $\Phi_{N, \boldsymbol{\lambda}}$ does not depend on $N$. 


\subsection{Proof of Lemma 1.4}

Lemma 2.1. For every $\boldsymbol{\lambda}$, the spectral variety $\operatorname{Spec}_{\boldsymbol{\lambda}} / S_{n} \subset T^{*}\left(\mathbb{C}^{n}-\Delta\right) / S_{n}$ is smooth. For different $\boldsymbol{\lambda}$ 's the spectral subvarieties do not intersect.

Proof. Let $x \in X_{\boldsymbol{\lambda}}^{0}$ and $\mathrm{Wr}_{\boldsymbol{\lambda}}(x)$ be a projection of $\boldsymbol{z}_{x}=\left(z_{1, x}, \ldots, z_{n, x}\right)$. By [18], the points $x \in X_{\lambda}^{0}$ are in a one-to-one correspondence with the eigenvectors of the Gaudin Hamiltonians on Sing $V^{\otimes n}[\boldsymbol{\lambda}]$. Denote $v_{x}$ the eigenvector corresponding to $x$. By Lemma 3.1 in [16] the numbers $\boldsymbol{p}_{x}=\left(p_{1, x}, \ldots, p_{n, x}\right)$ are eigenvalues of $H_{1}\left(\boldsymbol{z}_{x}\right), \ldots, H_{n}\left(\boldsymbol{z}_{x}\right)$ on $v_{x}$. Hence, the image of $\psi_{\boldsymbol{\lambda}}$ is $\operatorname{Spec}_{\boldsymbol{\lambda}} / S_{n}$.

By Theorem 3.2 in [16], the coordinates $z_{1, x}, \ldots, z_{n, x}, p_{1, x}, \ldots, p_{n, x}$ generate all functions on $X_{\boldsymbol{\lambda}}^{0}$. This proves that $\psi_{\boldsymbol{\lambda}}$ is an embedding of $X_{\boldsymbol{\lambda}}^{0}$ to $T^{*}\left(\mathbb{C}^{n}-\Delta\right) / S_{n}$.

By Theorem 3.2 in [16], the coefficients $P_{i j}(x)$ in (1.5) are given by some universal functions in $\boldsymbol{z}_{x}, \boldsymbol{p}_{x}$ independent of $\boldsymbol{\lambda}$. Hence formula (1.6) implies that the spectral varieties for different $\lambda$ 's do not intersect.

Lemma 2.2. For every $\boldsymbol{\lambda}$ the spectral variety $\mathrm{Spec}_{\boldsymbol{\lambda}}$ lies in the variety $L_{0}$ defined in (1.2).

Proof. Let $x \in X_{\boldsymbol{\lambda}}^{0}$ and $\boldsymbol{z}_{x}=\left(z_{1, x}, \ldots, z_{n, x}\right)$ corresponds to $\operatorname{Wr}_{\boldsymbol{\lambda}}(x)$. By Theorem 3.2 in [16],

$$
\operatorname{det}\left(\left(u-Z_{x}\right)\left(v-Q_{x}\right)-1\right)=\sum_{0 \leqslant i \leqslant j \leqslant n} P_{i j}(x) u^{n-j} v^{n-i}
$$

where $Z_{x}=\operatorname{diag}\left(z_{1, x}, \ldots, z_{n, x}\right), Q_{x}$ is given by (1.1) in terms of $\boldsymbol{z}_{x}$ and $\boldsymbol{p}_{x}$, and $P_{i j}(x)$ are given by (1.5). This implies that $\operatorname{det}\left(v-Q_{x}\right)=v^{n}$ and hence $\operatorname{Spec}_{\boldsymbol{\lambda}} \subset L_{0}$.

\subsection{Proofs of parts (i) and (ii) of Theorem 1.5, part $(\mathrm{ii})$ of Theorem 1.2, and Theorem 1.1}

Consider the Lie algebra $\mathfrak{g l}_{n}$ with standard generators $e_{i j}$. Fix a set of complex numbers $\boldsymbol{q}=$ $\left(q_{1}, \ldots, q_{n}\right)$. Consider the weight subspace

$$
V^{\otimes n}[1, \ldots, 1]=\left\{w \in V^{\otimes n} \mid e_{i i} w=w, i=1, \ldots, n\right\}
$$

and the Gaudin Hamiltonians

$$
H_{a}(\boldsymbol{z}, \boldsymbol{q})=\sum_{i=1}^{n} q_{i} e_{i i}^{(a)}+\sum_{i, j=1}^{n} \sum_{b \neq a} \frac{e_{i j}^{(a)} e_{j i}^{(b)}}{z_{a}-z_{b}},
$$

which generalize the Gaudin Hamiltonians in (1.3). The generalized Gaudin Hamiltonians act on $V^{\otimes n}[1, \ldots, 1]$. By Theorem 5.3 in [17], for generic $(\boldsymbol{z}, \boldsymbol{q})$ the generalized Gaudin Hamiltonians $H_{1}(\boldsymbol{z}, \boldsymbol{q}), \ldots, H_{n}(\boldsymbol{z}, \boldsymbol{q})$ have an eigenbasis in $V^{\otimes n}[1, \ldots, 1]$. By Theorem 4.3 in [15] the eigenvectors are in one-to-one correspondence with the preimages of the point $(\boldsymbol{z}, \boldsymbol{q})$ under the map $\pi: C_{n} \rightarrow \mathbb{C}^{(n)} \times \mathbb{C}^{(n)}$. This identification sends an eigenvector $w$ with $H_{a}(\boldsymbol{z}, \boldsymbol{q}) w=p_{a} w$, $a=1, \ldots, n$, to the point $(Z, Q)$, where $Z=\operatorname{diag}\left(z_{1}, \ldots, z_{n}\right)$ and $Q$ is defined by (1.1).

The $\mathfrak{g l}_{n}$-action on every eigenvector of the Gaudin Hamiltonians $H_{a}(\boldsymbol{z}, \boldsymbol{q}=0)$ on the space Sing $V^{\otimes n}[\boldsymbol{\lambda}]$ generates a $d_{\boldsymbol{\lambda}}$-dimensional subspace in $V^{\otimes n}[1, \ldots, 1]$ of eigenvectors of $H_{a}(\boldsymbol{z}, \boldsymbol{q}=0)$. The identification of Theorem 4.3 in [15] implies that $\operatorname{Spec}_{\boldsymbol{\lambda}}$ has multiplicity $d_{\boldsymbol{\lambda}}$ when defined by the equations $Q_{a}(\boldsymbol{z}, \boldsymbol{p})=0, a=1, \ldots, n$. More precisely, the identification tells that $d_{\boldsymbol{\lambda}}$ points of the $\pi^{-1}(\boldsymbol{z}, \boldsymbol{q})$ collide to one point of $\operatorname{Spec}_{\boldsymbol{\lambda}}$, when $\boldsymbol{q} \rightarrow 0$. This proves part (ii) of Theorem 1.5.

Since $\sum_{\boldsymbol{\lambda}} d_{\boldsymbol{\lambda}}^{2}=n$ !, we conclude that $L_{0}=\cup_{\boldsymbol{\lambda}} \operatorname{Spec}_{\boldsymbol{\lambda}}$. This proves part $(i)$ of Theorem 1.5, part (ii) of Theorem 1.2, and Theorem 1.1. 


\subsection{Proof of parts (iii) and (iv) of Theorem 1.5}

Every point of $X_{\boldsymbol{\lambda}}$ is a point of Wilson's adelic Grassmannian $\operatorname{Gr}^{\text {ad }}(n)$, which is identified with the Calogero-Moser space $C_{n}$ by the main Theorem 5.1 in [23]. For generic points of $X_{\boldsymbol{\lambda}}$ the map is $x \mapsto\left(Z_{x}, Q_{x}\right)$. The induced map of functions $\mathbb{C}\left[C_{n}\right] \rightarrow \mathbb{C}\left[X_{\boldsymbol{\lambda}}\right]$ is constructed as follows. Consider $P(u, v)=\operatorname{det}((u-Z)(v-Q)-1)$ as a polynomial in $u, v$ whose coefficients are functions on $C_{n}, P(u, v)=\sum_{i, j} \widetilde{P}_{i j} u^{n-j} v^{n-i}$. The coefficients $\widetilde{P}_{i j}$ generate $\mathbb{C}\left[C_{n}\right]$, see Lemma 4.1 in [15]. The map $\mathbb{C}\left[C_{n}\right] \rightarrow \mathbb{C}\left[X_{\lambda}\right]$ is defined by the formula $\widetilde{P}_{i j} \mapsto P_{i j}$, where $P_{i j}$ are given by (1.5), see Theorem 4.3 in [15]. By Lemma 3.4 in [18] the image of this map is $\mathbb{C}\left[X_{\boldsymbol{\lambda}}\right]$. Hence $X_{\boldsymbol{\lambda}} \rightarrow C_{n}$ is an embedding. By formula (1.6) the images do not intersect for different $\boldsymbol{\lambda}$ 's. This proves parts (iii) and (iv) of Theorem 1.5.

\section{Further remarks}

Fix distinct complex numbers $\boldsymbol{q}=\left(q_{1}, \ldots, q_{n}\right)$. Let $\sigma_{a}(\boldsymbol{q}), a=1, \ldots, n$, be the elementary symmetric functions of $\boldsymbol{q}$. Define

$$
L_{\boldsymbol{q}}=\left\{(\boldsymbol{z}, \boldsymbol{p}) \in T^{*}\left(\mathbb{C}^{n}-\Delta\right) \mid Q_{a}(\boldsymbol{z}, \boldsymbol{p})=\sigma_{a}(\boldsymbol{q}), a=1, \ldots, n\right\} .
$$

Theorem 3.1. The subvariety $L_{\boldsymbol{q}}$ is irreducible, smooth, and Lagrangian.

Define the spectral variety of the Gaudin Hamiltonians $H_{a}(\boldsymbol{z}, \boldsymbol{q}), a=1, \ldots, n$, on $V^{\otimes n}[1, \ldots, 1]$ by the formula

$$
\operatorname{Spec}_{\boldsymbol{q}}=\left\{(\boldsymbol{z}, \boldsymbol{p}) \in T^{*}\left(\mathbb{C}^{n}-\Delta\right) \mid \exists v \in V^{\otimes n}[1, \ldots, 1] \text { with } H_{a}(\boldsymbol{z}, \boldsymbol{q}) v=p_{a} v, a=1, \ldots, n\right\} .
$$

Theorem 3.2. We have $L_{\boldsymbol{q}}=\operatorname{Spec}_{\boldsymbol{q}}$.

Consider the set of $n(n-1) / 2$ variables

$$
\boldsymbol{t}=\left(t_{1}^{(1)}, \ldots, t_{n-1}^{(1)}, \ldots, t_{1}^{(n-2)}, t_{2}^{(n-2)}, t_{1}^{(n-1)}\right)
$$

and the affine space $\mathbb{C}^{n} \times \mathbb{C}^{n(n-1) / 2}$ with coordinates $\boldsymbol{z}, \boldsymbol{t}$. Consider the master function $\Phi_{\boldsymbol{q}}$ : $\mathbb{C}^{n} \times \mathbb{C}^{n(n-1) / 2} \rightarrow \mathbb{C}$,

$$
\begin{aligned}
\Phi_{\boldsymbol{q}}(\boldsymbol{z}, \boldsymbol{t})= & \sum_{1 \leqslant a<b \leqslant n} \log \left(z_{a}-z_{b}\right)-\sum_{a=1}^{n} \sum_{i=1}^{n-1} \log \left(t_{i}^{(1)}-z_{a}\right) \\
& +2 \sum_{k=1}^{n-1} \sum_{1 \leqslant i<j \leqslant n-k} \log \left(t_{i}^{(k)}-t_{j}^{(k)}\right)-\sum_{k=0}^{n-2} \sum_{i=1}^{n-k} \sum_{j=1}^{n-k-1} \log \left(t_{i}^{(k)}-t_{j}^{(k+1)}\right) \\
& +\sum_{k=1}^{n-1} \sum_{i=1}^{n-k}\left(q_{k+1}-q_{k}\right) t_{i}^{(k)}+q_{1} \sum_{a=1}^{n} z_{a},
\end{aligned}
$$

see [5]. Denote by $\mathrm{Crit}_{\boldsymbol{q}}$ the critical set of $\Phi_{\boldsymbol{q}}$ with respect to $\boldsymbol{t}$,

$$
\text { Crit }_{\boldsymbol{q}}=\left\{(\boldsymbol{z}, \boldsymbol{t}) \in \mathbb{C}^{n} \times \mathbb{C}^{n(n-1) / 2} \mid d_{\boldsymbol{t}} \Phi_{\boldsymbol{q}}(\boldsymbol{z}, \boldsymbol{t})=0\right\} .
$$

Denote by $\widetilde{L}_{\boldsymbol{q}} \subset T^{*}\left(\mathbb{C}^{n}-\Delta\right)$ the image of the map

$$
\mathrm{Crit}_{\boldsymbol{q}} \rightarrow T^{*}\left(\mathbb{C}^{n}-\Delta\right), \quad(\boldsymbol{z}, \boldsymbol{t}) \mapsto(\boldsymbol{z}, \boldsymbol{p}), \quad \text { where } \quad p_{a}=\frac{\partial \Phi_{\boldsymbol{q}}}{\partial z_{a}}(\boldsymbol{z}, \boldsymbol{t}), \quad a=1, \ldots, n .
$$


Theorem 3.3. We have $\widetilde{L}_{\boldsymbol{q}} \subset \operatorname{Spec}_{\boldsymbol{q}}$ and $\operatorname{Spec}_{\boldsymbol{q}}$ is the closure of $\widetilde{L}_{\boldsymbol{q}}$ in $T^{*}\left(\mathbb{C}^{n}-\Delta\right)$.

Consider the canonical map $\pi: C_{n} \rightarrow \mathbb{C}^{(n)} \times \mathbb{C}^{(n)},(Z, Q) \mapsto(\operatorname{spec}(Z), \operatorname{spec}(Q))$. Denote by $C_{n}^{\boldsymbol{q}}$ the subvariety $\pi^{-1}\left(\mathbb{C}^{(n)} \times \boldsymbol{q}\right) \subset C_{n}$. Identifying $\mathbb{C}^{(n)} \times \boldsymbol{q}$ with $\mathbb{C}^{(n)}$ we get a map $\pi^{\boldsymbol{q}}: C_{n}^{\boldsymbol{q}} \rightarrow \mathbb{C}^{(n)}$, $(Z, Q) \mapsto \operatorname{spec}(Z)$, induced by $\pi$.

Denote

$$
f_{i}(u)=e^{q_{i} u}\left(u+f_{i 1}\right), \quad i=1, \ldots, n .
$$

Denote by $X_{\boldsymbol{q}}$ the $n$-dimensional affine space of $n$-tuples $\left\{f_{1}, \ldots, f_{n}\right\}$ of such quasiexponentials. The polynomial algebra $\mathbb{C}\left[X_{\boldsymbol{\lambda}}\right]=\mathbb{C}\left[f_{11}, \ldots, f_{n 1}\right]$ is the algebra of regular functions on $X_{\boldsymbol{q}}$. We have

$$
\operatorname{Wr}\left(f_{1}(u), \ldots, f_{n}(u)\right)=e^{\left(q_{1}+\cdots+q_{n}\right) u} \prod_{1 \leqslant i<j \leqslant n}\left(q_{j}-q_{i}\right) \cdot\left(u^{n}+\sum_{a=1}^{n}(-1)^{a} W_{a} u^{n-a}\right)
$$

with $W_{1}, \ldots, W_{n} \in \mathbb{C}\left[X_{\boldsymbol{q}}\right]$. Define an algebra homomorphism

$$
\mathcal{W}_{\boldsymbol{q}}: \mathbb{C}\left[\mathbb{C}^{(n)}\right] \rightarrow \mathbb{C}\left[X_{\boldsymbol{q}}\right], \quad \sigma_{a} \mapsto W_{a}
$$

Let $\mathrm{Wr}_{\boldsymbol{q}}: X_{\boldsymbol{q}} \rightarrow \mathbb{C}^{(n)}$ be the corresponding map of spaces.

\section{Theorem 3.4.}

(i) The equations $Q_{a}=q_{a}, a=1, \ldots, n$, define $C_{n}^{\boldsymbol{q}}$ in $C_{n}$ with multiplicity 1.

(ii) There is an embedding $\varphi_{\boldsymbol{q}}: X_{\boldsymbol{q}} \rightarrow C_{n}^{\boldsymbol{q}}$ whose image is $C_{n}^{\boldsymbol{q}}$ and such that the following diagram is commutative:

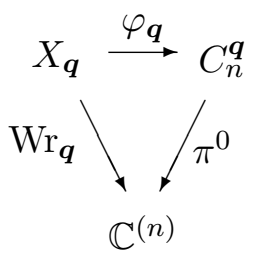

The map $\varphi_{\boldsymbol{q}}$ is given by the following construction. For $x=\left(f_{1}, \ldots, f_{n}\right) \in X_{\boldsymbol{q}}$, define the differential operator $\mathcal{D}_{\boldsymbol{q}, x}$ by

$$
\mathcal{D}_{\boldsymbol{q}, x}=e^{-\left(q_{1}+\cdots+q_{n}\right) u} \prod_{1 \leqslant i<j \leqslant n}\left(q_{j}-q_{i}\right)^{-1} \operatorname{rdet}\left(\begin{array}{cccc}
f_{1}(u) & f_{1}^{\prime}(u) & \ldots & f_{1}^{(n)}(u) \\
f_{2}(u) & f_{2}^{\prime}(u) & \ldots & f_{2}^{(n)}(u) \\
\ldots & \ldots & \ldots & \ldots \\
1 & \partial & \ldots & \partial^{n}
\end{array}\right) .
$$

Then

$$
\mathcal{D}_{\boldsymbol{q}, x}=\sum_{i=0}^{n} \sum_{j=0}^{n} P_{i j}(x) u^{n-j} \partial^{n-i}
$$

where $P_{i j} \in \mathbb{C}\left[X_{\boldsymbol{q}}\right]$.

Denote $X_{\boldsymbol{q}}^{0}=X_{\boldsymbol{q}} \cap \mathrm{Wr}_{\boldsymbol{\lambda}}^{-1}\left(\left(\mathbb{C}^{n}-\Delta\right) / S_{n}\right)$ and consider the map

$$
\psi_{\boldsymbol{q}}: X_{\boldsymbol{q}}^{0} \rightarrow T^{*}\left(\mathbb{C}^{n}-\Delta\right) / S_{n}, \quad x \mapsto\left(\boldsymbol{z}_{x}, \boldsymbol{p}_{x}\right),
$$


where $\boldsymbol{z}_{x} \in \mathbb{C}^{n}$ projects to $\mathrm{Wr}_{\boldsymbol{q}}(x) \in \mathbb{C}^{(n)}$ and $\boldsymbol{p}_{x}=\left(p_{1, x}, \ldots, p_{n, x}\right)$,

$$
p_{a, x}=-\operatorname{Res}_{u=z_{a, x}}\left(\frac{\sum_{j=0}^{n} P_{2, j}(x) u^{n-j}}{\prod_{i=1}^{n}\left(u-q_{i}\right)}\right)+\sum_{b \neq a} \frac{1}{z_{a, x}-z_{b, x}} .
$$

Then the restriction of $\varphi_{\boldsymbol{q}}$ to $X_{\boldsymbol{q}}^{0}$ is the composition $\xi \circ \psi_{\boldsymbol{q}}$, where $\xi$ is given by (1.4). This map extends from $X_{\boldsymbol{q}}^{0}$ to an embedding $X_{\boldsymbol{q}} \rightarrow C_{n}^{\boldsymbol{q}}$.

The proofs of Theorems 3.1-3.4 are basically the same as the proofs of Theorems 1.1-1.5.

\section{Acknowledgments}

The authors thank V. Schechtman and D. Arinkin for useful discussions. The third author thanks the MPIM, HIM, and IHES for the hospitality. E. Mukhin was supported in part by NSF grant DMS-0900984. V. Tarasov was supported in part by NSF grant DMS-0901616. A. Varchenko was supported in part by NSF grants DMS-0555327 and DMS-1101508.

\section{References}

[1] Babujian H.M., Off-shell Bethe ansatz equations and $N$-point correlators in the SU(2) WZNW theory, J. Phys. A: Math. Gen. 26 (1993), 6981-6990.

[2] Cherednik I., Integration of quantum many-body problems by affine Knizhnik-Zamolodchikov equations, Adv. Math. 106 (1994), 65-95.

[3] Cherednik I., Lectures on Knizhnik-Zamolodchikov equations and Hecke algebras, in Quantum Many-Body Problems and Representation Theory, MSJ Mem., Vol. 1, Math. Soc. Japan, Tokyo, 1998, 1-96.

[4] Etingof P., Ginzburg V., Symplectic reflection algebras, Calogero-Moser space, and deformed HarishChandra homomorphism, Invent. Math. 147 (2002), 243-348, math.AG/0011114.

[5] Felder G., Markov Y., Tarasov V., Varchenko A., Differential equations compatible with KZ equations, Math. Phys. Anal. Geom. 3 (2000), 139-177, math.QA/0001184.

[6] Felder G., Veselov A.P., Polynomial solutions of the Knizhnik-Zamolodchikov equations and Schur-Weyl duality, Int. Math. Res. Not. 2007 (2007), no. 15, 21 pages, math.RT/0610383.

[7] Felder G., Veselov A.P., Shift operators for the quantum Calogero-Sutherland problems via KnizhnikZamolodchikov equation, Comm. Math. Phys. 160 (1994), 259-273.

[8] Finkelberg M., Ginzburg V., Calogero-Moser space and Kostka polynomials, Adv. Math. 172 (2002), 137150, math.RT/0110190.

[9] Gaudin M., La fonction d'onde de Bethe, Collection du Commissariat à l'Énergie Atomique: Série Scientif ique, Masson, Paris, 1983.

[10] Givental A., Kim B., Quantum cohomology of flag manifolds and Toda lattices, Comm. Math. Phys. 168 (1995), 609-641, hep-th/9312096.

[11] Gordon I., Baby Verma modules for rational Cherednik algebras, Bull. London Math. Soc. 35 (2003), 321336, math.RT/0202301.

[12] Jurčo B., Classical Yang-Baxter equations and quantum integrable systems, J. Math. Phys. 30 (1989), 1289-1293.

[13] Kazhdan D., Kostant B., Sternberg S., Hamiltonian group actions and dynamical systems of Calogero type, Comm. Pure Appl. Math. 31 (1978), 481-507.

[14] Matsuo A., Integrable connections related to zonal spherical functions, Invent. Math. 110 (1992), 95-121.

[15] Mukhin E., Tarasov V., Varchenko A., Bethe algebra, Calogero-Moser space and Cherednik algebra, arXiv:0906.5185.

[16] Mukhin E., Tarasov V., Varchenko A., Gaudin Hamiltonians generate the Bethe algebra of a tensor power of the vector representation of $\mathfrak{g l}_{N}$, St. Petersburg Math. J. 22 (2011), 463-472, arXiv:0904.2131. 
[17] Mukhin E., Tarasov V., Varchenko A., Generating operator of $X X X$ or Gaudin transfer matrices has quasi-exponential kernel, SIGMA 3 (2007), 060, 31 pages, math.QA/0703893.

[18] Mukhin E., Tarasov V., Varchenko A., Schubert calculus and representations of the general linear group, J. Amer. Math. Soc. 22 (2009), 909-940, arXiv:0711.4079.

[19] Mukhin E., Varchenko A., Norm of a Bethe vector and the Hessian of the master function, Compos. Math. 141 (2005), 1012-1028, math.QA/0402349.

[20] Reshetikhin N., Varchenko A., Quasiclassical asymptotics of solutions to the KZ equations, in Geometry, Topology, \& Physics, Conf. Proc. Lecture Notes Geom. Topology, Vol. IV, Int. Press, Cambridge, MA, 1995, 293-322, hep-th/9402126.

[21] Schechtman V.V., Varchenko A.N., Arrangements of hyperplanes and Lie algebra homology, Invent. Math. 106 (1991), 139-194.

[22] Schechtman V.V., Varchenko A.N., Hypergeometric solutions of Knizhnik-Zamolodchikov equations, Lett. Math. Phys. 20 (1990), 279-283.

[23] Wilson G., Collisions of Calogero-Moser particles and an adelic Grassmannian, Invent. Math. 133 (1998), $1-41$. 